\title{
Synaptic Vesicle Transporter Expression Regulates Vesicle Phenotype and Quantal Size
}

\author{
Emmanuel N. Pothos, ${ }^{1}$ Kristin E. Larsen, ${ }^{1}$ David E. Krantz, ${ }^{3}$ Yong-jian Liu, ${ }^{4}$ John W. Haycock, ${ }^{6}$ Wanda Setlik, ${ }^{5}$ \\ Michael D. Gershon, ${ }^{5}$ Robert H. Edwards, ${ }^{3}$ and David Sulzer ${ }^{1,2}$ \\ 1 Departments of Neurology and Psychiatry, Columbia University, New York, New York 10032, 2Department of \\ Neuroscience, New York State Psychiatric Institute, New York, New York 10032, 3Departments of Neurology and \\ Physiology, University of California San Francisco School of Medicine, San Francisco, California 94143, ${ }^{4}$ Departments of \\ Neurology and Neuroscience, University of Pittsburgh School of Medicine, Pittsburgh, Pennsylvania 15261, ${ }^{5}$ Department \\ of Anatomy and Cell Biology, Columbia University, New York, New York 10032, and 'Department of Biochemistry and \\ Molecular Biology, Louisiana State University Medical Center, New Orleans, Louisiana 70119
}

While the transporters that accumulate classical neurotransmitters in synaptic vesicles have been identified, little is known about how their expression regulates synaptic transmission. We have used adenoviral-mediated transfection to increase expression of the brain vesicular monoamine transporter VMAT2 and presynaptic amperometric recordings to characterize the effects on quantal release. In presynaptic axonal varicosities of ventral midbrain neurons in postnatal culture, VMAT2 overexpression in small synaptic vesicles increased both quantal size and frequency, consistent with the recruitment of synaptic vesicles that do not normally release dopamine. This was confirmed using noncatecholaminergic AtT-20 cells, in which VMAT2 expression induced the quantal release of dopamine. The ability to increase quantal size in vesicles that were already competent for dopamine release was shown in PC12 cells, in which VMAT2 expression increased the quantal size but not the number of release events. These results demonstrate that vesicle transporters limit the rate of transmitter accumulation and can alter synaptic strength through two distinct mechanisms.

Key words: VMAT2; monoamines; dopamine; quantal size; amperometry; vesicular transporter
Transporters responsible for vesicular uptake of classical neurotransmitters from the cytoplasm belong to three distinct families, one for monoamines and acetylcholine, another for transmitters such as GABA (Reimer et al., 1998), and a third for glutamate (Bellochio et al., 2000). Inhibition or reduced activity of the vesicular transporters can decrease the amount of transmitter stored in vesicles, because both reduced quantal size and frequency has been observed at PC12 cells (Kozminski et al., 1998) and in a model of developing neuromuscular junction (Song and et al., 1997) after pharmacological blockade of uptake transport by reserpine or vesamicol. However, this effect is far less robust in mature neuromuscular junction, and only apparent after very prolonged bouts of stimulation (Van der Kloot et al., 2000). Mast cells, a non-neuronal secretory cell type, derived from mice with a single functional VMAT2 allele show a decreased number of serotonin and histamine molecules released per secretory event (Travis et al., 2000).

It is not known if native levels of transporter expression fill vesicles to equilibrium. Indeed, contemporary models of vesicle accumulation assume that the rate-limiting steps are determined by the electrochemical and concentration gradients across the vesicle membrane and that transporters are not rate-limiting (Johnson,

Received March 24, 2000; revised June 28, 2000; accepted July 17, 2000

This work was supported by a National Institute on Drug Abuse (NIDA) B/START award (E.N.P.), the Aaron Diamond Foundation (E.N.P.), Mr. and Mrs. John Z. Katz (E.N.P.), the National Alliance for Research on Schizophrenia and Depression (NARSAD) (E.N.P., Y.L., D.S.), a postdoctoral research fellowship for physicians from the Howard Hughes Medical Institute (D.E.K.), National Institutes of Health Grants NS25134 (J.W.H.), MH000967 (J.W.H.), NIDA 10154 (R.H.E., D.S.), NIDA 07418 (D.S.), and the Parkinson's Disease Foundation. E.N.P. is an Aaron Diamond Foundation Young Faculty Awardee and the Walter Sonneborn Katz Investigator funded by a 1999 NARSAD Young Investigator Award. We are grateful to Dr. Lloyd Greene, the late Dr. Menek Goldstein for providing cell lines, Dr. Steve Hardy for providing adenovirus and reagents, and Drs. Viviana Davila and Johanna Bogulavsky for expert technical assistance. We thank Serge Przedborski for critical reading of this manuscript.

Correspondence should be addressed to Dr. David Sulzer, Black Building, Room 305, 650 West 168th Street, New York, NY 10032. E-mail: ds43@columbia.edu. Copyright $(\odot 2000$ Society for Neuroscience $0270-6474 / 00 / 207297-10 \$ 15.00 / 0$
1988; Parsons et al., 1993; Sulzer and Pothos, 2000). However, overexpression of vesicular acetylcholine transporter (VAChT) in developing neuromuscular junction produces an increased amplitude of miniature EPSCs (Song et al., 1997). Whereas these immature synapses may posses low levels of transporter on vesicles, and postsynaptic changes such as receptor sensitization that could increase current cannot be ruled out, this suggests that native VAChT expression in this system does not completely fill vesicles.

Small synaptic vesicles in the CNS recycle and refill with neurotransmitter locally at the presynaptic site and maintain a tiny pool of releasable vesicles, estimated to be approximately four orders of magnitude smaller than at neuromuscular junction (Dobrunz and Stevens, 1997; Van der Kloot et al., 2000). Therefore, modulation of quantal size would have particularly important effects in the CNS. Mice that possess only one functional VMAT2 allele contain $\sim 50 \%$ of the dopamine and serotonin levels of wild-type animals (Fon et al., 1997; Takahashi et al., 1997; Wang et al., 1997). Ventral midbrain cultures derived from the ventral tegmental area (VTA) of these mice show a $50 \%$ reduction in depolarization-evoked dopamine release compared to wild-type cultures, suggesting either a reduced number of vesicles capable of storing transmitter or reduced quantal size because of decreased transmitter accumulation per vesicle (Fon et al., 1997).

To determine how increased vesicular transporter expression can regulate quantal release in a central preparation, we have used amperometric recordings at presynaptic sites on axons of cultured ventral midbrain neurons induced to overexpress VMAT2. Amperometry is the only extant method to directly measure the number of neurotransmitter molecules released per quantum, as well as provide the ability to resolve the kinetics of transmitter release during the exocytic event, and it does so without interference by alterations in postsynaptic receptors.

\section{MATERIALS AND METHODS}

Cell culture. AtT-20 cells including a line stably expressing tyrosine hydroxylase (TH) were a kind gift from the late Dr. Menek Goldstein (New York University) and Dr. John Haycock (Louisiana State University 
Medical School). TH immunolabel established that nearly all cells so transfected displayed TH protein (data not shown). AtT-20 cells were maintained in media containing 45\% Opti-MEM, 45\% DMEM, 5\% heatinactivated horse serum, $5 \%$ fetal bovine serum, and $50 \mathrm{U}$ each of penicillin and streptomycin. Experiments were conducted $3 \mathrm{~d}$ after plating.

PC12 cells obtained from Dr. Lloyd Greene (Columbia University) were plated at 40,000 cells per culture on glass coverslips coated with $7 \mathrm{ng} / \mathrm{mm}^{2}$ poly-D-lysine and maintained in Roswell Park Memorial Institute 1640 media supplemented with $10 \%$ heat-inactivated horse serum, $5 \%$ fetal bovine serum, and $50 \mathrm{U}$ each of penicillin and streptomycin. Cell lines were maintained at $35^{\circ} \mathrm{C}$ in $5 \%$ carbon dioxide. Experiments were conducted 7-14 d after plating.

VTA cultures were produced from day $0-2$ postnatal rats as reported (Burke et al., 1998; Pothos et al., 1998a) except that the cultures were maintained in medium containing 1\% calf serum (Rayport et al., 1992) supplemented at the time of plating with GDNF $(10 \mathrm{ng} / \mathrm{ml})$ to further promote axonal outgrowth and presynaptic varicosities (Burke et al., 1998). The use of $1 \%$ serum rather than serum-free media may underlie the small difference in average control quantal sizes in comparison to the previous study in this system; 7400 in the present study versus 11,400 in the earlier study (Pothos et al., 1998a). Recordings were conducted at 5-6 weeks after plating.

Viral transfection. For construction of adenoviral expression constructs, VMAT2 cDNA was subcloned into the pTet-A shuttle vector containing the cytomegalovirus $(\mathrm{CMV})$ promoter and upstream tet0 regulatory sites. For regulated expression of VMAT2 using Tet-A constructs, we used the helper virus TTA, which transactivates expression by binding to the tet 0 sites upstream of the CMV promoter; pTET-A-VMAT2 and TTA were cotransfected into human embryonic kidney (HEK) 293 cells. Tetracycline inhibits binding of TTA to tet 0 sites and was titrated to a concentration of $0.04 \mathrm{mg} / \mathrm{ml}$ to allow moderate levels of VMAT2 expression. The virus was purified using a $\mathrm{CsCl}$ step gradient and stored in $50 \%$ glycerol containing (in $\mathrm{mm}$ ): $10 \mathrm{Na} \mathrm{Pi}, 140 \mathrm{NaCl}$, and $4 \mathrm{KCl}, \mathrm{pH} \mathrm{7.4,} \mathrm{at} \sim 8000 \mathrm{pfu} / \mu \mathrm{l}$.

In addition, a hemagglutinin (HA)-tagged VMAT2 cDNA was first subcloned into the shuttle vector pTet-EF. pTet-EF-VMAT2 was then co-infected with donor virus DNA $(\Psi 5)$ into HEK293 cells expressing Cre recombinase (Cre 8 cells). This results in recombination between loxP sites in pTet-EF and $\Psi 5$ and the generation of an adenoviral construct containing VMAT2 cDNA and the viral backbone from 45 (Hardy et al., 1997). Expression from pTet-EF is driven by an EF1a promoter, and a moderate level of expression was observed in the absence of additional regulation. To purify recombinant virus, lysate from Cre8 HEK293 cells expressing recombinant VMAT2 was used to infect wild-type HEK293 cells, and virus was purified using a $\mathrm{CsCl}$ step gradient. Purified virus was stored as above. In some cases, Cre8 HEK293 cell lysate was used instead of purified virus.

For PC12 and AtT-20 cell recordings, cultures were incubated for $18 \mathrm{hr}$ at 1:1000 with either the TTA helper virus alone or in combination with pTet-A-VMAT2. The medium was replaced with fresh culture medium without virus $24 \mathrm{hr}$ before the experiments. Then, the cultures were washed and maintained in medium containing (in $\mathrm{mM}$ ): $150 \mathrm{NaCl}, 2 \mathrm{KCl}$, $1.2 \mathrm{CaCl}_{2}, 1 \mathrm{MgCl}_{2}, 1 \mathrm{NaH}_{2} \mathrm{PO}_{4}, 25$ glucose, and 10 HEPES, pH 7.3. The control cells received TTA virus alone.

Primary neuronal ventral tegmental area cultures at $30 \mathrm{~d}$ after plating were transfected by incubation for $2 \mathrm{hr}$ in a minimal volume of conditioned SF1C media containing a viral dilution of 1:100 PTet-EF-VMAT2. Cells were then gently washed twice with SF1C media and incubated for at least 10 additional days at a 1:2000 dilution. Neuronal toxicity was negligible using the Live/Dead assay (Molecular Probes, Eugene, OR; data not shown) (Cubells et al., 1994). Experiments were conducted at 5-6 weeks after plating.

Immunocytochemistry. For immunodetection of VMAT2, cultures were fixed in $4 \%$ paraformaldehyde in PBS, exposed to $0.1 \%$ Triton X-100 for $2 \mathrm{hr}$. We used a rabbit polyclonal antiserum generated against a peptide representing the $\mathrm{C}$ terminus of VMAT2 (Giorgio et al., 1995) (1:100, $48 \mathrm{hr}$ at $\left.4^{\circ} \mathrm{C}\right)$. A biotinylated goat anti-rabbit secondary antibody $(1: 200 ; 4 \mathrm{hr}$; $25^{\circ} \mathrm{C}$ ) and horseradish peroxidase-conjugated avidin-biotin complex with diaminobenzidine (Vectastain Elite kit; Vector Laboratories, Burlingame, CA) were used according to the manufacturer's instructions.

For light level immunodetection of HA-tagged recombinant VMAT2, cultures were fixed as above, exposed to $0.2 \%$ Triton X-100 in PBS with 5\% horse serum for $1 \mathrm{hr}$, and then to monoclonal anti-HA antibody (1:200; BabCo, Richmond, CA) in $0.2 \%$ Triton X-100 in PBS $\left(25^{\circ} \mathrm{C} ; 1 \mathrm{hr}\right)$. Fluorescent immunolabel used horse anti-mouse antibody conjugated to Texas Red (1:100; Vector Laboratories).

For immunodetection of synaptotagmin, cultures were fixed in $4 \%$ paraformaldehyde in DMEM at $4^{\circ} \mathrm{C}$, exposed to $0.2 \%$ Triton X-100 in PBS with $5 \%$ goat serum for $1 \mathrm{hr}$ and then to rabbit anti-rat lumenal epitope ( $\mathrm{N}$ terminus) synaptotagmin I (1:100; Alamone Labs) in $0.2 \%$ Triton $\mathrm{X}-100$ in PBS with $5 \%$ goat serum $\left(4^{\circ} \mathrm{C} ; 18 \mathrm{hr}\right)$. Fluorescent immunolabel used goat anti-rabbit antibody conjugated to FITC (1:100; Sigma, St. Louis, MO). Fluorescent photomicroscopy used a Photometrics Star 1 camera, and photoprocessing was performed with NIH Image.

For electron microscopic detection of HA-tagged recombinant VMAT2, cultures were fixed in PLP fixative (4\% paraformaldehyde with $77 \mathrm{~mm}$ lysine $\mathrm{HCl}, 10 \mathrm{~mm}$ sodium periodate, and $3 \%$ sucrose in $0.1 \mathrm{M}$ phosphate buffer, $\mathrm{pH}$ 7.4) for $15 \mathrm{~min}$. Fixed cells were washed in buffer and mor- danted with $0.25 \%$ tannic acid in $100 \mathrm{~mm}$ sodium phosphate buffer containing $3.5 \%$ sucrose for $1 \mathrm{hr}$ at $4^{\circ} \mathrm{C}$. Specimens were then quenched with $50 \mathrm{mM} \mathrm{NH}_{4} \mathrm{Cl}$ in the same buffer, washed extensively with $100 \mathrm{~mm}$ sodium maleate containing $4 \%$ sucrose, $\mathrm{pH} 6.2$, and stained en bloc with maleatebuffered $2 \%$ uranyl acetate. The stained tissues were dehydrated in the cold. Once the specimens were in $70 \%$ ethanol, the temperature was lowered to $-20^{\circ} \mathrm{C}$ for complete dehydration, clearing, and embedding in LR Gold (LR Gold Resin Company). LR Gold was polymerized by UV light at $-20^{\circ} \mathrm{C}$. Thin sections were cut and picked up on nickel grids that had been layered with Formvar. The sections were treated on the grids with a blocking solution containing $4 \%$ normal goat serum, $1.0 \%$ bovine serum albumin, and $0.5 \%$ cold-water fish gelatin in Tris-buffered saline for $30 \mathrm{~min}$ at room temperature. The sections were then incubated with a mouse monoclonal antibody to HA diluted 1:50 with blocking solution in which the normal serum concentration was reduced to $1 \%$. Grids were incubated overnight at $4^{\circ} \mathrm{C}$ and subsequently washed and incubated for $3 \mathrm{hr}$ at room temperature with goat anti-mouse antibody coupled to $10 \mathrm{~nm}$ particles of colloidal gold diluted 1:20 with blocking solution (Amersham, Arlington Heights, IL). The sections were washed, post-fixed with $2.5 \%$ glutaraldehyde in water for $5 \mathrm{~min}$, stained with $2 \%$ osmium, uranyl acetate, and lead citrate, and examined under a JEOL 1200 EX electron microscope.

Liquid chromatography. Dopamine levels were measured by HPLC-EC on an ESA (Bedford, MA) Coulochem II HPLC equipped with a model 5011 analytical cell with an applied potential of $400 \mathrm{mV}$ and a Velosep RP-18 column (Applied Biosystems, Foster City, CA). The mobile phase contained $6.9 \mathrm{gm} / 1 \mathrm{NaH}_{2} \mathrm{PO}_{4} \cdot \mathrm{H}_{2} \mathrm{O}, 80 \mathrm{mg} / 1$ EDTA $\cdot \mathrm{Na}_{2} \cdot 2 \mathrm{H}_{2} \mathrm{O}, 250$ $\mathrm{mg} / 1$ heptanesulfonic acid, and $6 \%$ methanol (adjusted to $\mathrm{pH} 3.6$ with phosphoric acid). To measure intracellular monoamine levels, medium was removed, and the cells were rapidly solubilized in $100 \mu \mathrm{l}$ of $0.3 \mathrm{M}$ perchloric acid. The samples were centrifuged at $15,000 \mathrm{rpm}$ for $15 \mathrm{~min}$ and $4^{\circ} \mathrm{C}$ and stored at $-80^{\circ} \mathrm{C}$ until HPLC analysis. Depolarization-dependent release in AtT-20 cells used normal incubation medium (see below) with $40 \mathrm{~mm} \mathrm{KCl}$ substituted equimolarly for $\mathrm{NaCl}\left(3 \mathrm{~min}, 37^{\circ} \mathrm{C}\right)$. The greater increase in dopamine release in VMAT2 overexpressing neurons observed with amperometry as compared to HPLC-EC is likely attributable to greater reuptake, metabolism, and depolarization inactivation over the longer period of time in the HPLC-EC protocols.

Detection of quantal release. Carbon fiber electrodes were constructed from $5 \mu \mathrm{m}$ carbon fibers (Amoco, Greenville, SC) in $1.2 \times 0.68 \mathrm{~mm}$ glass capillary tubes (A-M Systems, Everett, WA) pulled with a Flaming-Brown micropipette puller (Sutter Instruments, Novato, CA). The electrode tip was dipped into epoxy (Epo-Tek 301; Epoxy Technology, Billerica, MA) and cured at $100^{\circ} \mathrm{C}$ for $24 \mathrm{hr}$. Electrodes were back-filled with $3 \mathrm{M} \mathrm{KCl}$. Electrode tips were polished at $40^{\circ}$ on a beveller (World Precision Instruments, New Haven, CT). Electrode response was tested by cyclic voltammetry in freshly prepared nitrogen-bubbled $10 \mu \mathrm{M}$ dopamine solution, and those with unstable $I-V$ curves or high rms noise $(>1 \mathrm{pA}$; Axon Instruments; three-pole $10 \mathrm{kHz}$ Butterworth filter) were rejected. The electrode was placed at the recording site with a Huxley-style micropositioner (Newport Instruments, Irvine, CA). A $+700 \mathrm{mV}$ voltage versus $\mathrm{Ag}-\mathrm{AgCl}$ ground was applied to the carbon fiber electrode using an Axon 200A amplifier (Axon Instruments). The output was digitized at $50 \mathrm{kHz}$ for PC12 and AtT-20 cells and low-pass filtered at $10 \mathrm{kHz}$ using a four-pole Bessel filter. PC12 data were digitally refiltered at $10 \mathrm{kHz}$ (GWI Instruments, Medford, MA). The population characteristics of the rapid events in AtT-20 cells in Table 1 are reported without the $10 \mathrm{kHz}$ digital filter, because this rolled off the amplitude of the more rapid component of the hybrid event (see Fig. $2 J$ ). Amplitude roll off of longer-duration events was negligible, and events shown in Figure 2 are not digitally filtered.

For CNS neurons, the output was digitized at $160 \mathrm{kHz}$ and low-pass filtered at $10 \mathrm{kHz}$ using a four-pole Bessel filter in the Axopatch $200 \mathrm{~A}$ amplifier. Before analysis, the current wave was digitally refiltered using a smoothing function at $8 \mathrm{kHz}$. Whereas increased filtering uncovered significantly more events in recordings from dispersed acutely dissociated retinal amacrine cells (Hochstetler et al., 2000), in our preparation we observed few such extra events of sufficient amplitude to reach the $5.0 \times$ rms noise threshold with filtering as low as $1 \mathrm{kHz}$ (data not shown).

For electrochemical recordings, normal incubation medium contained (in mM): $150 \mathrm{NaCl}, 2 \mathrm{KCl}, 1.2 \mathrm{CaCl}_{2}, 1 \mathrm{MgCl}_{2}, 1 \mathrm{NaH}_{2} \mathrm{PO}_{4}, 25$ glucose, and $10 \mathrm{HEPES}, \mathrm{pH}$ 7.3. High $\mathrm{KCl}$ stimulation medium was composed of (in $\mathrm{mM}$ ): $72 \mathrm{NaCl}, 80 \mathrm{KCl}, 6 \mathrm{CaCl}_{2}, 21$ glucose, and 10 HEPES, pH 7.3. Because VTA neurons are recorded at sites of small $(\sim 1-\mu$ m-diameter axonal varicosities and typically exhibit very few events after exposure to high $\mathrm{KCl}$, we used the potent secretagogue $\alpha$-latrotoxin in VTA cultures to promote a higher number of amperometric events (Pothos et al., 1998a). $\alpha$-Latrotoxin stimulation medium contained $20 \mathrm{nM} \alpha$-latrotoxin (a kind gift of Alexander Petrenko, New York University) in addition to the above.

In all cases, cells were stimulated by 3 or $6 \mathrm{sec}$ applications of $\sim 20 \mathrm{nl}$ volumes of stimulation medium at a distance of $18 \mu \mathrm{m}$ from the recorded cell (Picospritzer; General Valve, Fairfield, NJ). Low-pressure ( $<7-8$ psi) was applied to avoid mechanical stimulation, so that we never observed electrochemical spikes during perfusion with physiological medium.

Amperometric data analysis. Quantal events were analyzed using a locally written Superscope II program (GW Instruments). The average baseline current in the vicinity of the spikes was subtracted from the signal. Spikes were identified if the amplitudes were 4.5 times (AtT-20 and PC12 cells) or 
5.0 times (VTA neurons) greater than the rms background noise. The number of molecules (quantal size) oxidized at the electrode face was determined by the relation $N=Q / n F$, where $Q$ is the charge of the spike, $n$ is the number of electrons transferred per molecule [shown to be two for catecholamines when used in a similar experimental configuration $(\mathrm{Ci}$ olkowski et al., 1994)], $N$ is the number of moles, and $F$ is Faraday's constant $(96,485$ coulombs per equivalent) (for a more thorough discussion of this issue, see Sulzer and Pothos, 2000). The following additional parameters were measured for each spike: the number of molecules in the foot (foot molecules) [the foot is identified as the portion of the event preceding the slope as defined by the angle of the $60-90 \%$ incline of the maximal rising slope (Chow and von Ruden, 1995), $t_{1 / 2}$ (the width at half maximal amplitude), and the maximal amplitude $\left(i_{\max }\right)$. For AtT-20 cells, to clearly delineate the subpopulations of events, we also determined the duration, defined as the time between the start of the upward slope and the first point at which the decay reaches the baseline.

Ribonuclease protection assay. RNA was isolated and purified using the Totally RNA Total RNA Isolation kit (Ambion, Austin, TX). Briefly, cells were lysed on the culture dish after a rinse with $1 \times \mathrm{PBS}$, and then homogenized by centrifugation in a QiaShredder spin column (Qiagen Santa Clarita, CA) at $13,000 \times g$ for $30 \mathrm{sec}$. The homogenate was subjected to two rounds of phenol-chloroform extraction followed by overnight isopropanol precipitation. RNA samples were pelleted by centrifugation $(13,000 \times g$ for $15 \mathrm{~min})$, washed with $70 \%$ ethanol, resuspended in RNase-free water-0.1 M EDTA, digested with $10 \mathrm{U}$ of RNase-free DNase I, and extracted first by phenol-chloroform, then by chloroform-isoamyl alcohol. The DNA-free RNA was collected by ethanol precipitation, and the quantity of the RNA was determined spectrophotometrically.

Ribonuclease protection assay (RPA) kits (HybSpeed RPA; Ambion) and in vitro transcription kits (Maxiscript, Ambion) were used following the manufacturer's instructions. Radiolabeled RPA probes for TH, VMAT2, and $\beta$-actin were prepared from cDNAs (200-350 nucleotides) by in vitro transcription. Briefly, $1 \mu \mathrm{l}$ of cDNA was incubated in Maxiscript buffer containing $1 \mathrm{mM}$ ATP, GTP, and TTP, $10 \mu \mathrm{M}$ UTP, $3 \mu \mathrm{M}\left[{ }^{32} \mathrm{P}\right] \mathrm{UTP}$, RNase inhibitor, and T7 or SP6 RNA polymerase $(2000 \mathrm{U} / \mu \mathrm{l})$ at $37^{\circ} \mathrm{C}$ for $1 \mathrm{hr}$, then at $95^{\circ} \mathrm{C}$ for $5 \mathrm{~min}$, then placed on ice. DNase was then added to the reaction for $15 \mathrm{~min}$ at $37^{\circ} \mathrm{C}$. The unincorporated label was removed, and the specific activity of each probe was determined by liquid scintillation counting. For hybridization, $1 \times 10^{5} \mathrm{cpm}$ of probe was hybridized with $0.5-1 \mu \mathrm{g}$ of neuronal RNA sample or with $50 \mu \mathrm{g}$ of yeast total RNA (RPA control) in HybSpeed hybridization buffer at $95^{\circ} \mathrm{C}$ for $3 \mathrm{~min}$, followed by incubation at $68^{\circ} \mathrm{C}$ for $10 \mathrm{~min}$. After hybridization, $100 \mu \mathrm{l}$ of HybSpeed RNase digestion buffer/RNase A/T1 was added to each sample, mixed, and incubated at $48^{\circ} \mathrm{C}$ for $1 \mathrm{hr}$. Then $150 \mu \mathrm{l}$ of HybSpeed inactivation-precipitation mix, $50 \mu \mathrm{l}$ of $100 \%$ ethanol, and $0.5 \mu \mathrm{l}$ of glycoblue was added to each sample, vortexed, and chilled at $-20^{\circ} \mathrm{C}$ overnight. The precipitated products were pelleted by centrifugation at $13,000 \times g$ for 15 min, the pellets were resuspended in RPA gel loading buffer, and loaded onto a nondenaturing 5\% acrylamide gel, and electrophoresed for $3 \mathrm{hr}$ at $100 \mathrm{~V}$. The gel was then visualized using a PhosphoImaging screen.

Western blotting. After exposure to adenovirus, cells were washed with PBS and lysed with $300 \mu \mathrm{l}$ of $10 \mathrm{~mm}$ Tris-EDTA, pH 7.5, containing $1 \%$ SDS, $4 \% \beta$-mercaptoethanol, glycerol, and bromophenol blue. After scraping, the samples were boiled for $5 \mathrm{~min}$, sonicated to disrupt genomic DNA, and centrifuged at $13,000 \times g$ for $1 \mathrm{~min}$. Ten micrograms of total protein were loaded and separated by $8 \%$ SDS-PAGE on $8 \%$ gels, transferred to nitrocellulose membrane (Bio-Rad, Hercules, CA), and probed with a mouse anti-TH monoclonal antibody (1:640 dilution; Boehringer Mannheim, Indianapolis, IN). For visualization, a biotinylated horse anti-mouse secondary antibody (1:200 dilution; Vector Laboratories) for $1 \mathrm{hr}$, followed by an avidin-biotin-HRP complex for $1 \mathrm{hr}$ was used, and the antibody complex was visualized using DAB and hydrogen peroxide.

\section{RESULTS}

\section{TH expression induces rare quantal dopamine release in AtT-20 cells}

To determine whether VMAT2 expression is sufficient to enable dopamine release from a noncatecholaminergic cell type, we used mouse-derived adrenocorticotroph AtT-20 cells, a non-neuronal secretory cell type that displays regulated release, most prominently of dense core granules. Wild-type AtT-20 cells express an endogenous aromatic acid decarboxylase capable of converting L-DOPA to dopamine (Horellou et al., 1989) but do not express mRNA for TH (Fig. 1a), VMAT1 (data not shown), or VMAT2 (Fig. 1a).

No catecholamines or metabolites were detected by HPLC with electrochemical detection (EC) in wild-type AtT-20 cells (data not shown), and no amperometric events were detected from over 30 wild-type AtT-20 cells recorded after stimulation with high-K ${ }^{+}$ medium ( $80 \mathrm{~mm}, 6 \mathrm{sec})$. To determine if depolarization can stimulate release in the presence of cytosolic dopamine but without VMAT2, we used AtT-20 cells stably expressing TH (Horellou et al., 1989; Harada et al., 1996). These cells released dopamine under stimulation with high $\mathrm{K}^{+}(40 \mathrm{~mm})$, as measured by HPLC-EC (Fig. $1 b)$. No other monoamines or catecholamine metabolites (homovanillic acid or dihydroxyphenylacetic acid) were present in either intracellular or extracellular samples.

To measure dopamine release from individual secretory vesicles, we recorded from cell bodies using 5- $\mu \mathrm{m}$-diameter carbon fiber amperometric microelectrodes (Wightman et al., 1991). This technique provides kinetic data on quantal release at an order of magnitude higher time resolution than postsynaptic recordings, which are limited by the kinetics of postsynaptic receptors. In addition, the charge measured during amperometric recording is directly related to the number of molecules released per quanta, independent of receptor saturation or desensitization.

AtT-20 cell transformants expressing only recombinant TH displayed very rare amperometric events after stimulation with $80 \mathrm{~mm}$ $\mathrm{K}^{+}$(Fig. $2 a, c ; n=9$ from 49 cells recorded, $1.0 \pm 0.0$ events per cell from the cells that showed release). Therefore, a very small number of neurosecretory vesicles apparently accumulate and release detectable levels of dopamine in the absence of a VMAT. Diffusion of dopamine followed by trapping of the protonated transmitter in the acidic vesicles may account for exocytic release in the absence of VMAT activity.

\section{VMAT2/TH coexpression promotes vesicular dopamine release}

To induce VMAT2 expression, we used a recombinant adenovirus encoding rat VMAT2. Infection by the adenovirus resulted in VMAT2 expression, as detected by immunostaining with a polyclonal antibody to VMAT2 (Fig. $3 a, b$ ). Immunoreactivity was present throughout the cytoplasm but excluded from nuclei. VMAT2 mRNA was also detected in the recombinant adenovirusinfected AtT-20 cells (Fig. 1a).

Two hours after exposure to the recombinant adenovirus, cultures expressing both TH and VMAT2 released 90\% more dopamine after stimulation $\left(50 \mathrm{~mm} \mathrm{~K}{ }^{+}, 6 \mathrm{sec}\right)$ than cells transfected with TH alone (Fig. $1 b ; p<0.01$; one-way ANOVA). The intracellular dopamine level was similarly elevated (Fig. $1 c ; p<0.01$ ). After infection for $12 \mathrm{hr}$, dopamine release was increased by $280 \%$, the maximum level detected. No other monoamines or catecholamine metabolites were detected in either intracellular or extracellular samples.

The increased levels of dopamine observed could reflect upregulation of TH protein by VMAT2. However, at 2, 4, 6, 12, 24, and 48 hr after infection with the VMAT construct, TH protein levels were not different from either control cultures or cultures treated with helper adenovirus alone (Fig. 1d).

VMAT2/TH expression increased the number of evoked quantal events by 4.4-fold over AtT-20 cells expressing only TH (Tables $1,2 ; p<0.0001$; Student's $t$ test; Fig. $2 a, b)$. The mean quantal size for all events was also increased to $320 \%$ of control levels ( $p=$ 0.03; Kolmogorov-Smirnov test $(\mathrm{KS}-Z)=1.4482)$.

We observed two major populations of amperometric events that differed in duration (Fig. $2 c-n$ ). The more rapid events displayed a duration of $<1 \mathrm{msec}$, faster than that associated with dense core vesicles, but similar to results in neurons with small clear synaptic vesicles (Bruns and Jahn, 1995; Pothos et al., 1998a; Hochstetler et al., 2000). This bimodal population can be seen in histograms as in Figure $2 g$, which shows a single large bin for events with $<10,000$ molecules and a non-normal distribution of larger sizes. If the log transforms of the values are displayed (Fig. $2 h$ ) it appears that there are two close-to-normal distributions corresponding to the apparent two populations in the untransformed histogram. A similar bimodal distribution can be seen for the event durations, in which again a log transform yields close-to-normal distributions (Fig. $2 i, j$ ).

As has been shown previously for quantal analysis at the neuromuscular junction (Van der Kloot, 1991) and explained in detail in a recent review (Sulzer and Pothos, 2000), bimodal distributions can be more clearly observed using a normal probability distribu- 
Table 1. Characteristics of quantal events elicited from TH- and VMAT2-expressing AtT-20 cells

Molecules released

$\mathrm{i}_{\max }(\mathrm{pA})$

$t_{1 / 2}(\mathrm{msec})$

Duration (msec)

\begin{tabular}{|c|c|c|c|c|}
\hline \multicolumn{5}{|l|}{ All quanta } \\
\hline $\mathrm{TH}$ & $12,600 \pm 4300$ & $23.3 \pm 11.3$ & $1.9 \pm 1.0$ & $3.55 \pm 1.5$ \\
\hline VMAT2/TH & $40,100 \pm 2900^{*}$ & $7.7 \pm 1.4$ & $4.1 \pm 0.2$ & $11.7 \pm 0.7$ \\
\hline VMAT2/TH/L-DOPA & $76,000 \pm 14,700^{\#}$ & $33.6 \pm 6.2^{\# \# \#}$ & $3.2 \pm 0.5$ & $11.1 \pm 2.4$ \\
\hline \multicolumn{5}{|l|}{ Duration $<1 \mathrm{~ms}$} \\
\hline $\mathrm{TH}$ & $6300 \pm 2310$ & $41.3 \pm 22.0$ & $0.06 \pm 0.01$ & $0.13 \pm 0.02$ \\
\hline VMAT2/TH & $11,700 \pm 3200$ & $45.4 \pm 13.8$ & $0.15 \pm 0.05$ & $0.37 \pm 0.08$ \\
\hline VMAT2/TH/L-DOPA & $5600 \pm 1600$ & $69.1 \pm 10.2$ & $0.08 \pm 0.02$ & $0.13 \pm 0.01^{\#}$ \\
\hline \multicolumn{5}{|l|}{ Duration $>1 \mathrm{~ms}$} \\
\hline $\mathrm{TH}$ & $25,600 \pm 6700$ & $9.8 \pm 7.8$ & $3.3 \pm 1.4$ & $7.2 \pm 2.2$ \\
\hline VMAT2/TH & $42,100 \pm 3000^{*}$ & $5.5 \pm 1.0$ & $4.2 \pm 0.2$ & $12.4 \pm 0.8$ \\
\hline VMAT2/TH/L-DOPA & $118,400 \pm 22,100^{\# \# \#}$ & $7.2 \pm 3.1^{\# \# \#}$ & $5.5 \pm 0.6^{\# \# \#}$ & $18.6 \pm 3.5^{\# \# \#}$ \\
\hline
\end{tabular}

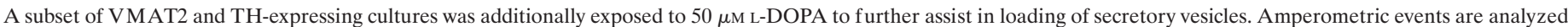

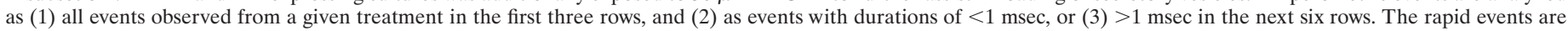
reported without digital filtering (see Materials and Methods). The $n$ of events in the categories are listed in Table 2.

${ }^{*}$ Different from TH by Kolmogorov-Smirnov; $p<0.05$, ${ }^{* *} p<0.01$, ${ }^{* * *} p<0.001$.

${ }^{\#}$ Different from VMAT2/TH by Kolmogorov-Smirnov; $p<0.05,{ }^{\# \#} p<0.01,{ }^{\# \# \# p}<0.001$.

a
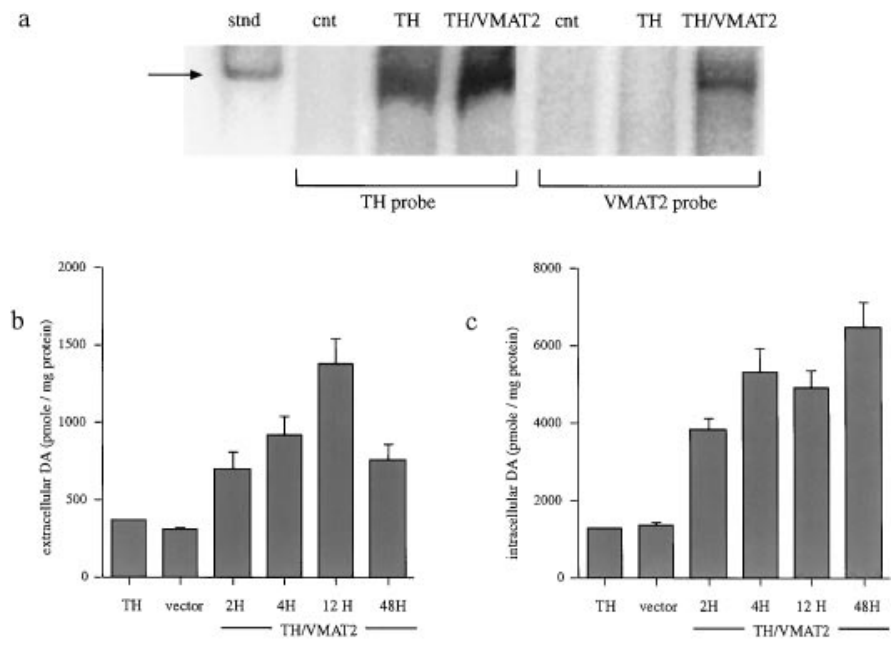

d

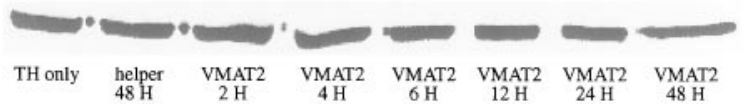

Figure 1. Effect of VMAT2 and TH cotransfection in AtT-20 cells. $a$, Ribonuclease protection assays indicate that message for $\mathrm{TH}$ is present only in stably transfected lines and that VMAT2 message is present only in adenoviral-exposed cultures. The recombinant genes are labeled at the top of each column, and the probes used are bracketed on the bottom of each column. The standard (arrow) in the leftmost column indicates a band of $258 \mathrm{kDa}$, close to the expected fragment lengths. $\beta$-actin was present in all cultures at similar levels (data not shown). $b, c$, Cultures transfected with TH only, or cotransfected with TH and VMAT2 (2-48 hr), or with TH and control virus (vector), were exposed to $40 \mathrm{mM} \mathrm{K}{ }^{+}$for $3 \mathrm{~min}$ at $37^{\circ} \mathrm{C}$. Released (extracellular) $(b)$ and remaining intracellular $(c)$ dopamine levels were measured. Mean \pm SEM are indicated, $n=3$ cultures per group. No dopamine was observed in cultures that did not express $\mathrm{TH}$, and no extracellular dopamine was observed in unstimulated preparations (data not shown). The experiment was repeated three times with similar results. $d$, Western blotting for TH protein showed no significant differences between controls, helper virus treated cultures, and VMAT2 expressors. Ten micrograms of protein were loaded per lane. The bands show TH protein from left to right in control, helper virus only, and 2, 4, 6, 12, 24, and $48 \mathrm{hr}$ after exposure to the adenovirus. The experiment was performed three separate times, and VMAT2 expression in each case was functionally confirmed before Western blotting (using the protocols in $b$ and $c$ ). Densitometry measurements normalized for control levels were as follows: helper virus only for $48 \mathrm{hr}, 94 \pm 9 \% ; 2 \mathrm{hr}, 94 \pm 9 \% ; 4 \mathrm{hr}, 94 \pm 6 \% ; 6 \mathrm{hr}, 96 \pm 9 \%$; $12 \mathrm{hr}, 97 \pm 2 \% ; 24 \mathrm{hr}, 92 \pm 12 \% ; 48 \mathrm{hr}, 76 \pm 28 \%($ mean $\pm \mathrm{SD} ; n=3 ; F=$ $0.7141 ; p>0.1$; one-way ANOVA). tion in which each data point is plotted as a function of SDs from the mean. In this plot, a normal distribution is a straight line. For untransformed quantal sizes (Fig. $2 k$ ) and durations (Fig. $2 m$ ), the plots show obvious deviation from a normal distribution. The log transforms of the quantal sizes (Fig. 2l) and durations (Fig. 2n) show that at least two normally distributed populations are present.

While the rapid events were uncommon (4\% of events), their incidence increased greatly with exposure to L-DOPA (Table $2 ; p<$ 0.001), which has been shown to effectively load transmitter in catecholaminergic vesicles (Pothos et al., 1996, 1998a; Mena et al., 1998). Interestingly, the rapid events did not show an increased quantal size with L-DOPA but showed an increased incidence (Table 2), suggesting that some of these vesicles loaded only with high levels of cytosolic dopamine.

\section{VMAT2 expression increases quantal size in PC12 cells}

To determine whether VMAT2 overexpression in vesicles that contain an endogenous transporter increases transmitter accumulation, we used the pheochromocytoma PC12 cell line. PC12 cells express the neurosecretory VMAT subtype VMAT1 and release two classical transmitters, dopamine (and/or norepinephrine) from large dense core granules and acetylcholine from small clear vesicles (Bauerfeind et al., 1993; Liu et al., 1994; Liu and Edwards, 1997; Kozminski et al., 1998). Under the conditions used in this study in which cells are not exposed to nerve growth factor, nearly all of the neurotransmitter is released from large dense core vesicles. To induce VMAT2 expression, we used the adenoviral vector encoding VMAT2. Immunostain with a VMAT2 antibody showed no reactivity in control PC12 cells, whereas transfected cells were strongly labeled (Fig. 3c,d).

Quantal release from PC12 cell bodies was measured by amperometry (Chen et al., 1994; Sulzer et al., 1995). Expression of recombinant VMAT2 increased the quantal size in PC12 cells by fourfold (Fig. 4, Table 3; KS-Z = 5.1183; $p<0.001$ ), with corresponding increases in amplitude, duration, and width at half height for the transfected population (Table 3). However, the distribution of interspike intervals was not altered, suggesting that in wild-type, nondifferentiated PC12 cells, all the vesicles that release dopamine already express VMAT2. The quantal sizes closely fit unimodal lognormal distributions $\left(r^{2}=0.994, p<0.01\right.$ and $r^{2}=0.976, p<$ 0.01 for control and VMAT2 transfectants, respectively). Additional controls infected with nonrecombinant helper virus lacking the VMAT2 coding sequence did not exhibit enhanced dopamine release. 
a.
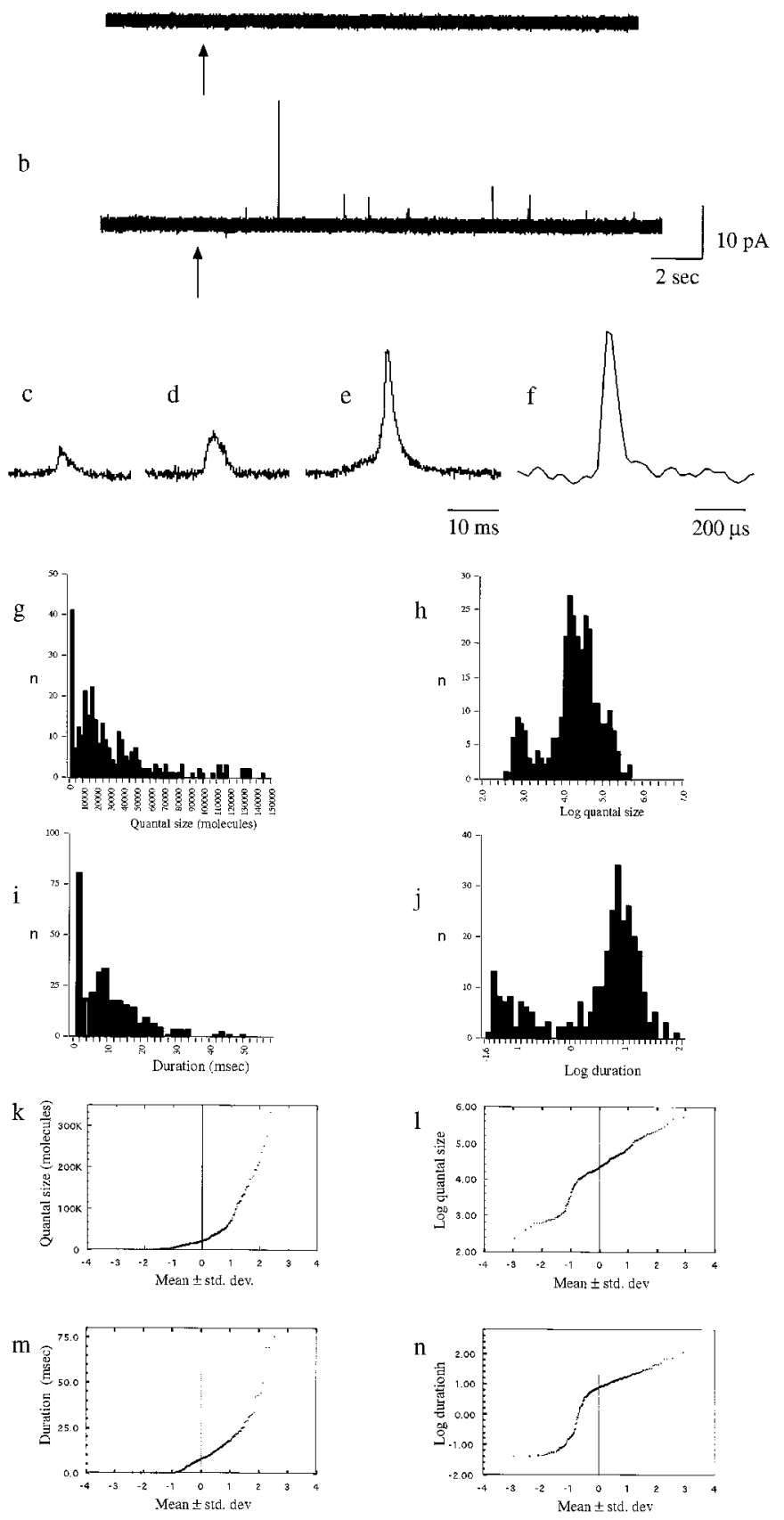

Figure 2. Effects of VMAT2 cotransfection on amperometric recordings of quantal release from TH-expressing AtT-20 cells. Traces of recordings from a representative TH-only cell $(a)$ and a VMAT2/TH-cotransfected cell with nine release events $(b)$ are shown. The arrows indicate the starting point of an application of $40 \mathrm{mM} \mathrm{K}^{+}(3 \mathrm{sec})$. Examples are shown of a rare wide duration peak from a TH-only culture $(c)$, a representative event from a VMAT2/TH cotransfected culture $(d)$, and an event from a cotransfected culture exposed to L-DOPA $(100 \mu \mathrm{M}$ for $30 \mathrm{~min})(e) . f$ indicates a sample rapid event from a cotransfected culture. $g$ indicates a histogram of the quantal sizes of all AtT-20 cell amperometric events recorded. Note that the smallest quantal sizes appear to belong to a separate distribution from the rest of the population. In $h$, the data in $k$ is plotted as a log transformation. The small size population can now be seen to have a close-tolognormal population distribution, similar in pattern but with a lower occurrence than the wider population. $i$ indicates a histogram of the durations of all AtT-20 amperometric events recorded. For this distribution as well, the most rapid events appear to be separate from the rest of the population. A log transformation of the data in $j$ indicates the presence of two populations. The data in $g-j$ is also plotted as normal probability plots in traces $k-n$. The points on the graph represent each event in the data. On the $x$-axis, " 0 " represents the mean value, and other values represent SDs from the mean. In this form, a normal distribution is represented by a straight line. The histograms and log transforms of the data are consistent with at least two separate populations of quantal events.
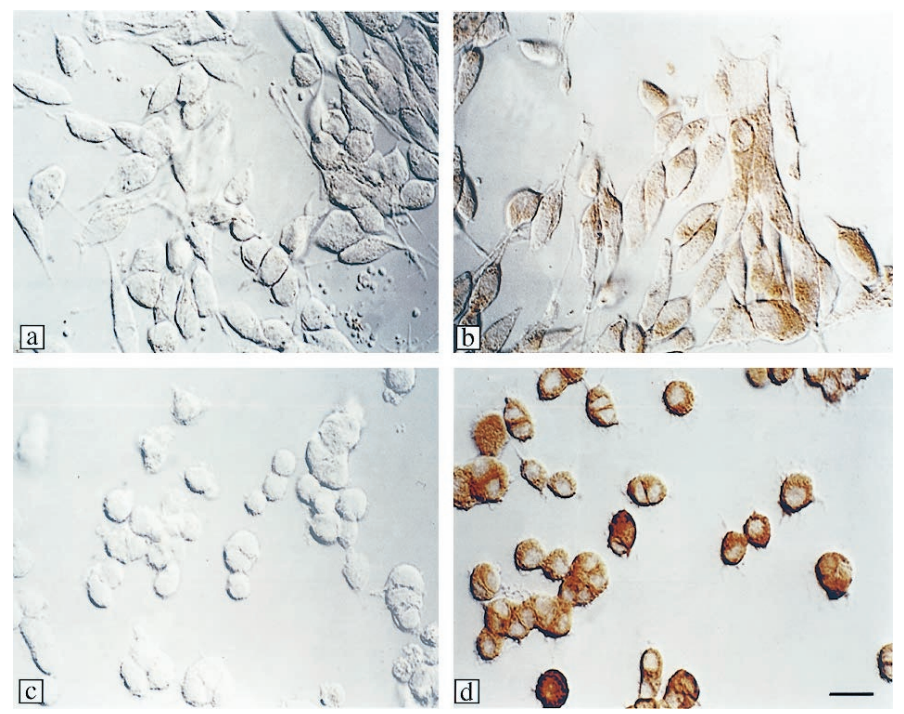

Figure 3. Immunoreactivity to VMAT2, as indicated by diaminobenzidine reaction product, in a TH-transfected AtT-20 culture $(a)$, a $\mathrm{TH}-$ and VMAT2-coexpressing AtT-20 culture $(b)$, a control PC12 culture $(c)$, and a VMAT2-transfected PC12 culture $(d)$. Scale bar: $a, b, 32 \mu \mathrm{m} ; c, d, 20 \mu \mathrm{m}$.

\section{VMAT2 overexpression in ventral midbrain neurons}

VTA cultures derived from neonatal rats contain $\sim 40 \%$ dopamine neurons based on TH immunoreactivity (Rayport and Sulzer, 1995; Przedborski et al., 1996). The remainder appear GABAergic because they exhibit immunoreactivity for glutamic acid decarboxylase. Adenoviral exposure did not detectably alter the morphology of these neurons, although exposure for longer than that used in this study damaged astrocytes.

The midbrain dopamine neurons in culture develop axons of $>1$ $\mathrm{mm}$ in length that display prominent axonal varicosities. These varicosities are filled with small (40- to 50-nm-diameter) synaptic vesicles and rare (typically $<2 \%)$ small $(\sim 100$-nm-diameter) dense core vesicles, as shown in our previous studies on this midbrain culture system (Sulzer and Rayport, 1990; Rayport et al., 1992; Pothos et al., 1998a). The varicosities often exist in isolation from apparent postsynaptic targets, which allows amperometric recording electrodes to be placed sufficiently close $(<300 \mathrm{~nm})$ to measure quantal dopamine release from the varicosities (Sulzer and Pothos, 2000).

Because midbrain dopamine neurons express endogenous VMAT2, we included an HA epitope in the VMAT2 cDNA to distinguish the recombinant protein from the native transporter. HA immunoreactivity after adenoviral infection was observed with a wide distribution within cell bodies and neurites, but not in nuclei. Approximately $20 \%$ of neurons were unlabeled for HA, and glial expression was generally absent. We used double labeling with the synaptic vesicle marker synaptotagmin-1 to identify sites that possess synaptic vesicles. As expected, synaptotagmin-1 immunolabel was highly specific for axonal varicosities. We found extensive colocalization of synaptotagmin with recombinant VMAT2 at axonal varicosities (Fig. $5 a-c$ ), but not at other sites.

We have previously published electron micrographs of these axonal varicosities in cultured dopaminergic VTA neurons (Sulzer and Rayport, 1990; Rayport et al., 1992; Pothos et al., 1998a). In the present study, we found that HA immunoreactivity was not retained after glutaraldehyde fixation (data not shown) but that immunoreactivity was preserved using PLP fixative. Whereas this fixative does not provide ultrastructural preservation as well as glutaraldehyde, numerous 40- to 50-nm-diameter small synaptic vesicles can be observed in presynaptic axonal varicosities. The location, size, and distribution of these vesicles was identical to those displayed in our previous studies using conventional fixation protocols. In the presynaptic axonal varicosities, HA-tagged recombinant VMAT2 was localized on small synaptic vesicle membranes 
Table 2. Population of quantal events measured from AtT-20 cells

\begin{tabular}{lllcrr} 
& $\begin{array}{l}\mathrm{N} \\
\text { cells }\end{array}$ & $\begin{array}{l}\% \text { cells } \\
\text { released }\end{array}$ & $\begin{array}{l}\text { \# quanta } \\
(n)\end{array}$ & Events/stim & $\%>1 \mathrm{msec}$ \\
\hline TH & 49 & $18 \%$ & 9 & $1 \pm 0$ & $5(56 \%)$ \\
VMAT2/TH & 80 & $43 \%$ & 201 & $4.39 \pm 0.53^{*}$ & $187(93 \%)$ \\
VMAT2/TH/L-DOPA & 36 & $50 \%$ & 82 & $5.76 \pm 0.98^{*}$ & $30(37 \%)$
\end{tabular}

Characteristics of spike size and shapes of these events are reported in Table 1. The number of cells recorded per condition $(\mathrm{N}$ cells), the percentage of the cells that showed amperometric events (\% cells released), the total number of events per category (\# quanta), the mean \pm SEM of amperometric events per stimulation for those cells that displayed release (events/stim), and the percentage of events with duration $>1 \mathrm{msec}(\%>1 \mathrm{msec})$ are indicated.

*Different from control, $p<0.0001, t$ test.

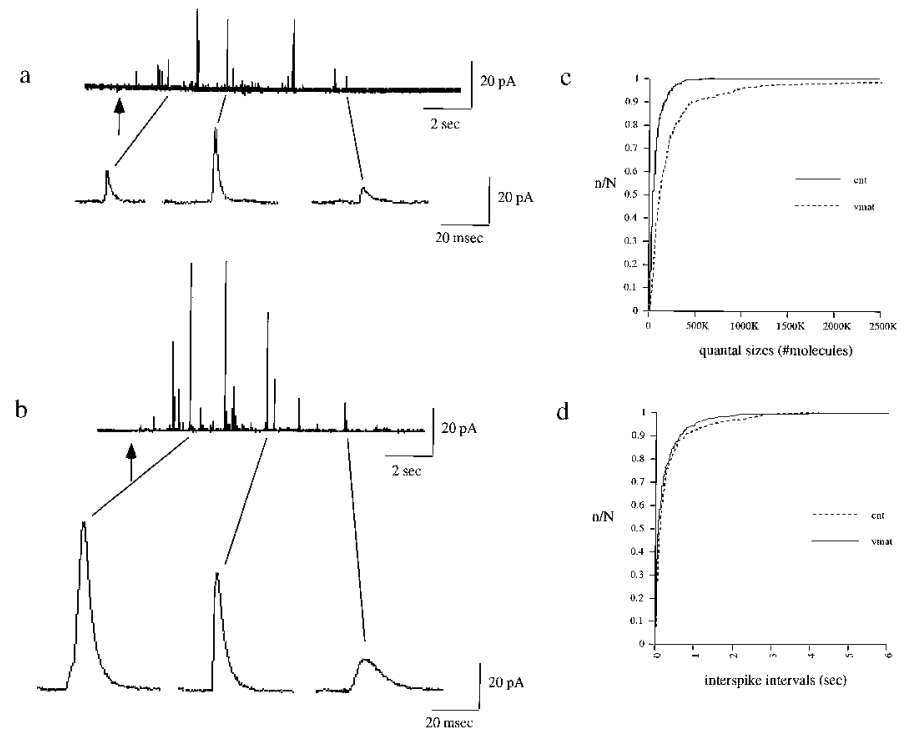

Figure 4. Representative examples of amperometric data contrasting release from control $(a)$ and VMAT2-transfected $(b)$ PC12 cells. The traces shown display a mean quantal size close to the population average. Examples of individual quanta are shown at an expanded time scale. The control trace displays 25 events with mean amplitude of $11.8 \mathrm{pA}$ and mean quantal size of 73,100 molecules. The VMAT2-transfected trace displays 25 events with mean amplitude of $17.8 \mathrm{pA}$ and mean quantal size of 329,900 molecules. $c, d$, The cumulative populations of quantal sizes and interspike intervals as shown in Table 3.

using anti-HA antibody coupled to $10 \mathrm{~nm}$ gold particles (Fig. $5 d-f$ ). We did not observe any immunogold label on dense core vesicles in synaptic terminals, although it is possible that the very low presence of dense cores was not sufficient to detect the presence of the antigen.

To compare total dopamine and dopamine releasable after stimulation in VMAT2-overexpressing cultures, we measured released dopamine and the remaining intracellular pool by HPLC-EC after exposure to $40 \mathrm{~mm} \mathrm{KCl}$ for $3 \mathrm{~min}$. Stimulated dopamine release increased to $246 \%$ of control levels in recombinant VMAT2expressing cultures relative to uninfected cultures (Fig. 6). The remaining intracellular dopamine in recombinant VMAT2expressing cultures also increased to $148 \%$ of control levels; VMAT2 transfection increased total dopamine per culture by $171 \%(p<0.1$, Newman-Keuls, including combined basal extracellular, releasable, and intracellular levels).

Consistent with the measurements of total release, overexpression of VMAT2 increased quantal release of dopamine. The events displayed the kinetic parameters associated with small synaptic vesicle exocytosis (Bruns and Jahn, 1995; Pothos et al., 1998a; Hochstetler et al., 2000). The increased release involved both an increase in quantal size (Table 4 , Fig. $7 a-d$; KS- $Z=3.4591 ; p<$ 0.001 ) and the frequency of events evoked by stimulation, as indicated by decreased interspike intervals (Fig. $7 e$; KS- $Z=2.541 ; p<$ $0.001)$. Overexpression of VMAT2 potentiated dopamine release in four independent sets of ventral midbrain cultures.
The increase in quantal size did not result from the increased frequency of release, which could potentially produce overlapping events consisting of multiple quanta. If many simultaneous but independent quanta were recorded in the transfected group, a Poisson distribution would be expected, reflecting multiples of single quanta. However, when plotted as a normal probability plot (see above), the distribution of the log transforms of the quantal sizes appears as nearly a straight line. Therefore, we conclude that the $\log$ values of the quantal sizes showed a unimodal normal distribution (Fig. $7 d, r^{2}=0.977$ from a straight line, $p<0.01$ for controls; $r^{2}=0.991, p<0.01$ for transfectants), rather than a Poisson distribution. Such a normal distribution of the log transforms of the quantal sizes has been found for quantal population distributions in other neurons tested (Van der Kloot, 1991; Pothos et al., 1998a).

Thus, there is a unimodal population of quantal sizes in this preparation. Because both the control and overexpressing groups show linear distributions, it appears that the entire population of quanta in the VMAT2 overexpressing neurons displays increased quantal size.

The frequency of stochastic simultaneous release events recorded from the transfected neurons can be estimated from the fusion probability distribution function determined by measuring latency times between consecutive amperometric spikes (Alvarez de Toledo and Fernandez, 1990). A close fit to an exponential decay is consistent with independent events. From the single exponential decay of the interspike intervals [Fig. $7 F: y=(0.8472)$ $e^{(-0.005 * t)} ; r^{2}=0.950$; ANOVA, $\left.F=14263, p<0.01\right]$ with time constant $\tau=1 / 0.005=200 \mathrm{msec}$, the probability of finding two events that would occur within an interval $t$ (here $t=1 \mathrm{msec}$ ) is given by:

$$
1-P(t)=1-e^{-t / \tau}=\sim 0.005 .
$$

This frequency is far too low to explain the increased quantal size. The very low fraction of overlapping events in these recordings despite strong stimulation is not surprising. A very small number of release sites (one to three) are recorded during amperometric measurements from axonal varicosities (Pothos et al., 1998a), whereas conventional postsynaptic recordings at cell bodies detect input from tens or hundreds of release sites.

\section{DISCUSSION}

We find that elevated vesicular transporter expression strongly regulates neurotransmitter release by CNS neurons. This regulation involves two distinct mechanisms: (1) recruitment of synaptic vesicles that previously contained no transporter allows vesicular accumulation with an attendant higher frequency of release, and (2) enhanced expression in vesicles already expressing a transporter elevates transmitter accumulation per vesicle, resulting in an increased quantal size. This would result in the detection of more quantal events, an increase in release frequency, and a decrease in interspike interval.

Together, these effects underlie the most robust plastic change in presynaptic function yet reported by altered protein expression in a CNS preparation. We find that whereas quantal sizes from 
Table 3. Characteristics of quantal events from control and VMAT2-transfected PC12 cells

\begin{tabular}{|c|c|c|c|c|c|}
\hline & Molecules released & Foot molecules & $i_{\max }(\mathrm{pA})$ & $t_{1 / 2}(\mathrm{msec})$ & $i_{\text {spike }}(\mathrm{msec})$ \\
\hline Control & $73,500 \pm 5500$ & $7400 \pm 500$ & $9.29 \pm 0.53$ & $2.53 \pm 0.10$ & $321 \pm 33$ \\
\hline VMAT2 & $283,400 \pm 36,900$ & $12,300 \pm 800$ & $17.53 \pm 1.40$ & $3.25 \pm 0.11$ & $253 \pm 31$ \\
\hline VMAT/Control (\%) & $385 \% * * *$ & $167 \%$ & $188 \% * * *$ & $129 \% * * *$ & $79 \%$ \\
\hline
\end{tabular}

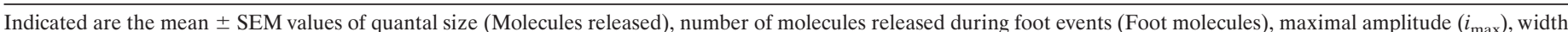

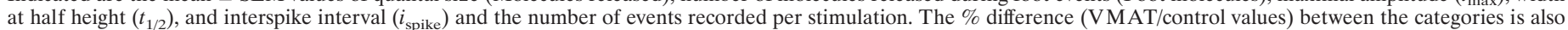
noted. The control group represents 346 events from 9 cells and the VMAT2 group consists of 349 events from 11 cells.

${ }^{* * *} p<0.001$, Kolmogorov-Smirnov statistics.
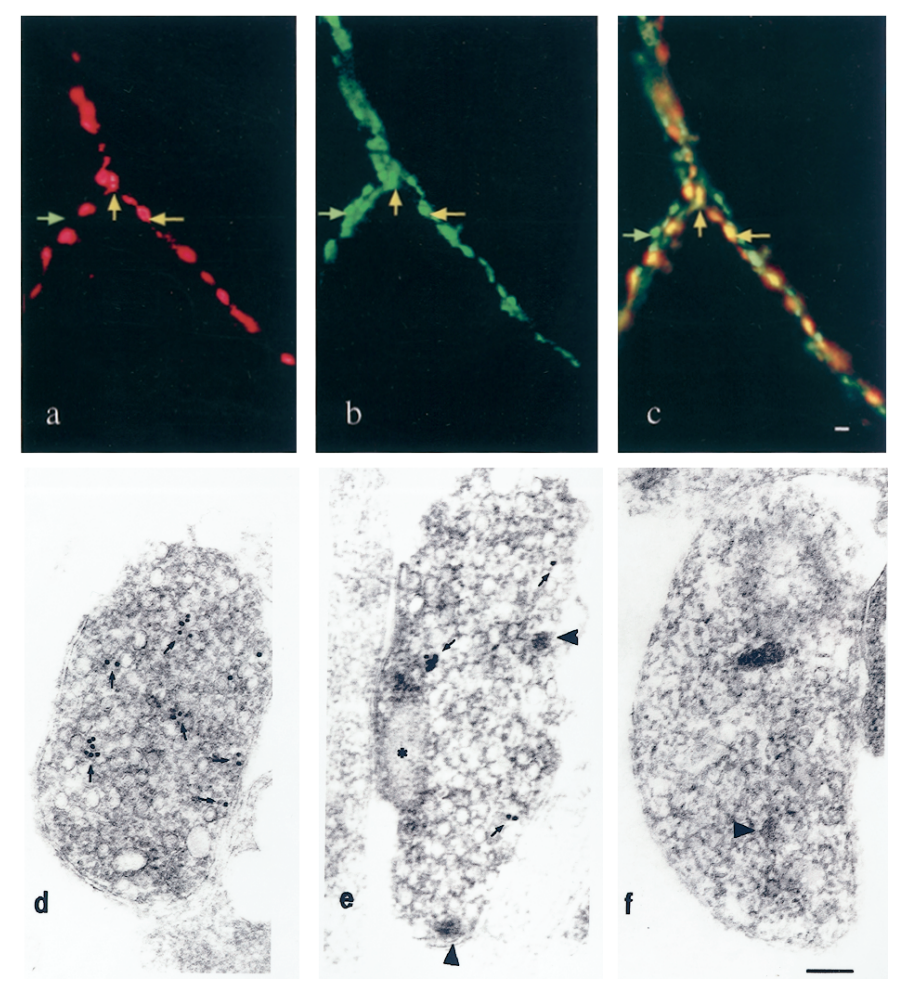

Figure 5. Immunolabel of recombinant VMAT2 in VTA neurons. $a$, Immunolabel for recombinant VMAT2 (red) in axons in an infected culture. $b$, Immunolabel of endogenous synaptotagmin-1 (green). c, Combined image of $a$ and $b$. Colocalization of both antigens appears yellow. Two examples of varicosities that express both recombinant VMAT2 and synaptotagmin are indicated by yellow arrows. An example of a varicosity that expresses synaptotagmin but not recombinant VMAT2 is indicated by a green arrow. Untransfected cultures or cultures exposed to a control adenovirus that does not contain the recombinant VMAT2 are not immunolabeled for recombinant VMAT2 (data not shown). Scale bar, $1 \mu \mathrm{m} . d, e$, Electron micrographs of PLP-fixed synaptic vesicles in axonal varicosities immunolabeled for recombinant VMAT2. The distribution of small synaptic vesicles, which can be recognized as 40 - to 50 -nm-round profiles, is identical to that seen in conventionally fixed micrographs in our previous publications (see Results). More than 40 small synaptic vesicle profiles are present in each of the terminals. The small synaptic vesicle membranes are immunopositive for recombinant VMAT2, as indicated by $10 \mathrm{~nm}$ gold particles (small arrows). Immunolabel is absent in dense core granules (large arrowheads). The asterisk indicates a mitochondria within a presynaptic terminal. Dendrites closely apposed to the terminals are not immunoreactive. $f$, Control axon terminals where the primary antibody was omitted are immunonegative, as are cultures not exposed to the adenovirus or to a control adenovirus that does not contain the recombinant VMAT2 (data not shown). Scale bars, $100 \mathrm{~nm}$.

wild-type neurons tend to exhibit a relatively narrow population, VMAT2 overexpression elicits some stimulation-dependent quantal events that release 10 -fold more dopamine than controls. While the mean increase in quantal size in VTA neurons is 2.6-fold, this probably underestimates the actual increase in quantal size because VMAT2 overexpression may also produce small amperometric spikes that would otherwise fall below limits of detection in the wild-type. In addition to increased quantal size, in VTA neurons

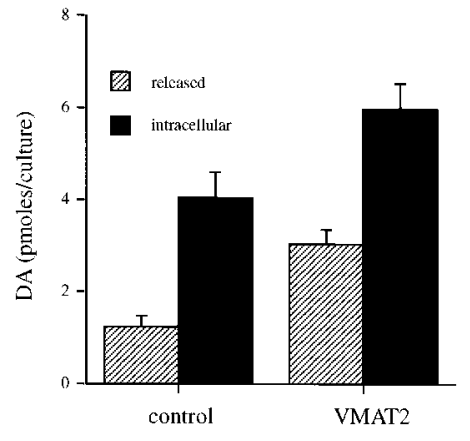

Figure 6. Effect of VMAT2 cotransfection on dopamine expression and release in VTA neurons. Stimulation-dependent dopamine release $(50 \mathrm{~mm}$ $\left.\mathrm{K}^{+}, 3 \mathrm{~min}\right)$ and remaining intracellular levels $(p<0.01$; Newman-Keuls test, for both released and intracellular dopamine levels; $n=7)$.

there is a 2.6-fold increase in the frequency of quantal events after stimulation. In contrast, synaptic strength in long-term potentiation paradigms often shows an increase of $\leq 1.5$-fold, with postsynaptic recordings indicating a mean increase in quantal size of similar levels in those instances that it occurs at all (Kullmann and Nicoll, 1992).

To confirm the two distinct mechanisms by which elevated transporter expression potentiates neurotransmitter release, we also induced novel expression of VMAT2 in two neurosecretory cell lines. Overexpression in the catecholaminergic PC12 cell line, which normally possesses large dense core granules that express VMAT1, increases quantal size but not frequency. Expression in the normally noncatecholaminergic AtT-20 cell lines enables them to release dopamine in a quantal manner.

\section{Increased VMAT2 activity elevates quantal size}

We propose that increased transporter expression recruits transmitter uptake-competent and fusion-competent but unfilled synaptic vesicles to accumulate more transmitter. VMAT2 overexpression shifted the entire unimodal population of events to larger values in both VTA neurons and PC12 cells.

The events in VTA neurons are attributable to exocytosis of small synaptic vesicles, because they are recorded from axonal varicosities that are filled with small synaptic vesicles and a very low presence $(<2 \%)$ of large dense core vesicles (Sulzer and Rayport, 1990; Rayport et al., 1992; Pothos et al., 1998a). In the present study, we show that these sites express recombinant VMAT2 on small synaptic vesicle membrane. Previous studies show that presynaptic axonal varicosities display endogenous VMAT2 (Nirenberg et al., 1997; Pothos et al., 1998a) and recycling compartments as demonstrated using the endocytic tracer FM1-43 (Pothos et al., 1998a). Moreover, the rapid time course of the release events has been observed only with small synaptic vesicles in neurons (Bruns and Jahn, 1995; Pothos et al., 1998a; Hochstetler et al., 2000), and is two or three orders of magnitude more rapid than that from dense core vesicles. A very small subpopulation of quantal events at the axonal varicosities resemble exocytosis from large dense core granules (Pothos et al., 1998a); however, no such events were recorded in the experiments in the present study.

In PC12 cells, the prolonged duration of quantal events is con- 
Table 4. Characteristics of quantal events recorded from control and VMAT2-transfected VTA neurons from sister cultures recorded over a $24 \mathrm{hr}$ period

\begin{tabular}{|c|c|c|c|c|}
\hline & Molecules released & $i_{\max }(\mathrm{pA})$ & $t_{1 / 2}(\mu \mathrm{sec})$ & $i_{\text {spike }}(\mathrm{msec})$ \\
\hline Control & $7400 \pm 700$ & $7.96 \pm 0.51$ & $270 \pm 23$ & $1645 \pm 321$ \\
\hline VMAT2 & $19,300 \pm 800$ & $12.42 \pm 0.24$ & $816 \pm 50$ & $630 \pm 57$ \\
\hline VMAT/Control (\%) & $261 \% * * *$ & $156 \% * * *$ & $302 \% * * *$ & $38 \% * * *$ \\
\hline
\end{tabular}

Indicated are the mean \pm SEM values of quantal size (Molecules released), maximal amplitude $\left(i_{\max }\right)$, width at half height $\left(t_{1 / 2}\right)$, and mean interspike interval $\left(i_{\text {spike }}\right)$. The \% difference (VMAT2/control values) between the categories is noted. The control group represents 88 events from eight exposures to $\alpha$-latrotoxin, and the VMAT2 group consists of 975 events from nine exposures.

*** $p<0.001$, Kolmogorov-Smirnov statistics.

a
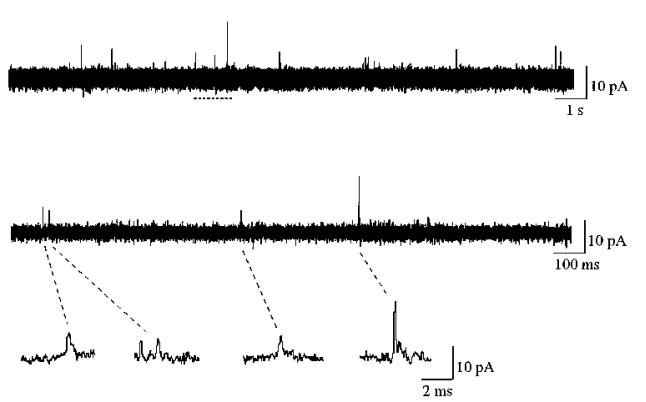

c

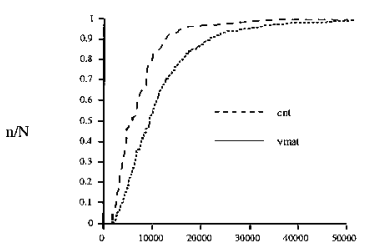

quantal sizes (fimolecules)

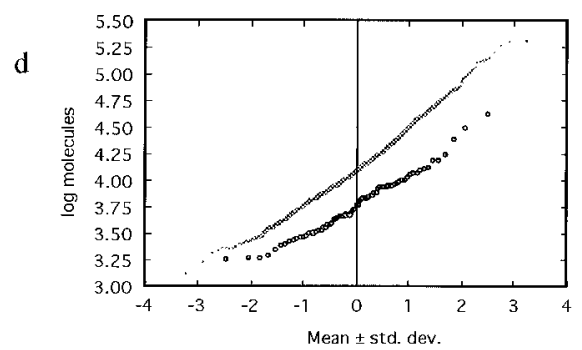

b
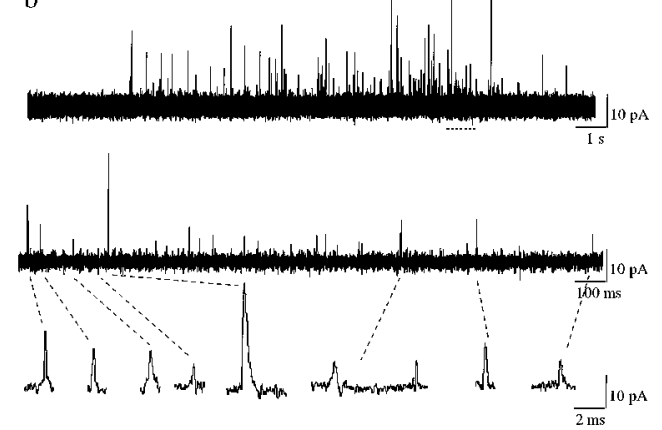

e
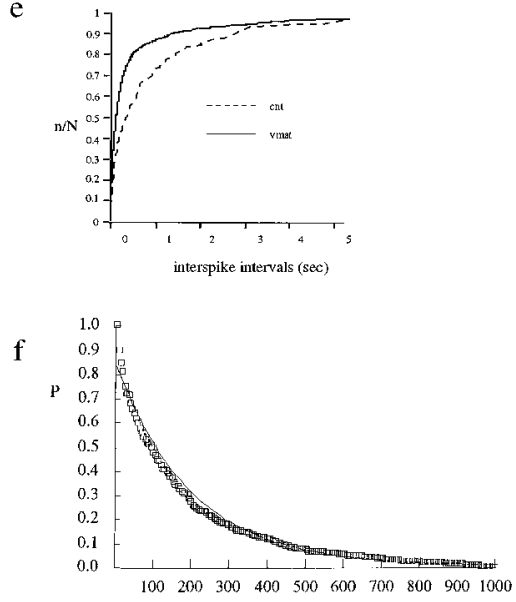

VMAF 2 interspike intervals (nswc)

Figure 7. Effect of VMAT2 cotransfection on quantal release in VTA neurons. Examples of amperometric data contrasting release from neurites in control $(a)$ and VMAT2-transfected $(b)$ cells after exposure to $20 \mathrm{nM} \alpha$-latrotoxin $(3 \mathrm{sec})$. The portion indicated by the broken lines is expanded in the middle trace. Examples of these events as marked by the second array of broken lines are displayed at increased temporal resolution in the discontinuous traces. The top control trace displays 12 events (with amplitudes $>5 \times$ rms background). These have a mean amplitude of $8.92 \mathrm{pA}$ and mean quantal size of 7800 molecules. The VMAT2-transfected trace displays 113 events with a mean amplitude $11.40 \mathrm{pA}$ and mean quantal size of 11,800 molecules. The distribution of quantal sizes is shown in $c$ and represents the events in Table 4. $d$ indicates the quantal sizes as a normal probability plot (Fig. 2, legend). On the $x$-axis, " 0 " represents the mean value, and other values represent SDs from the mean. The $y$-axis reports the log transforms of the quantal sizes. Data from the transfected cultures are in the top trace (smaller points), and controls are in the bottom trace (hollow points). The log transforms of the data are consistent with a different unimodal distribution of quanta in each group; compare Figure $2 L$ for an example of multimodal distribution. The distribution of interspike intervals for control cultures and cultures expressing recombinant VMAT2 are shown in $e$ and represent the events in Table 4. $f$ displays the probability distribution function of interspike intervals in the transfected group, which is well fit to a near exponential decay. All intervals in the data of $<1000$ msec are binned in $5 \mathrm{msec}$ intervals (diamonds). The best-fit exponential decay, determined by the least-squares method, is shown by the solid line.

sistent with exocytosis of large dense core granules (Chen et al., 1994). Therefore, VMAT2 overexpression can regulate quantal size both in small synaptic vesicles that recycle within CNS axonal varicosities as well as large dense core vesicles, which presumably recycle membrane in the cell body and require peptide refilling at the Golgi apparatus.

A normal distribution of the log transform of the number of dopamine molecules released per quantum is consistent with a distribution determined by multiplicative deviations from the mean, as would occur with relatively few transporters per vesicle (Van der Kloot, 1991; Pothos et al., 1998b). Regulation of a small number of transporters per vesicle could take place by the specific trafficking of transporters to different synaptic vesicles, by stochastic distribution of small numbers of transporters among the vesicles (Krantz et al., 1997), or by regulation of activity by second messengers. Indeed, recent evidence suggests regulation of activity of VMAT1 by G-proteins in permeabilized PC12 cells (AhnertHilger et al., 1998), and there is indirect evidence for VMAT1 modulation by cAMP (Nakanishi et al., 1995). In CNS axon terminals, regulation of transporter activity would play an important role in modulating the rate of transmitter refilling during the synaptic vesicle cycle. 
Whereas quantal size is usually thought to be invariant, presynaptic recordings have recently demonstrated alterations including elevation in quantal size after growth factor exposure or elevated transmitter synthesis (Pothos et al., 1998b) and a decrease in quantal size after collapse of electrochemical gradients (Sulzer et al., 1995) or a reduction in cytoplasmic transmitter (Pothos et al., 1998a,b). However, these mechanisms are all consistent with ratelimiting roles played only by the electrochemical and concentration gradients (Johnson, 1988). The present study suggests that endogenous vesicle transporter expression is rate-limiting. This could occur if there is a leak of transmitter from the vesicles, so that an increased number of transporters would maintain a higher fill rate. An endogenous leak of neurotransmitter has previously been shown in isolated synaptic vesicle fractions (Floor et al., 1995). The present evidence suggests that this leak also occurs within the neuron.

\section{VMAT2 activity can convert the vesicle neurochemical phenotype}

The evidence that there is a genuine conversion of synaptic vesicle phenotype is most clearly seen in the increase in dopaminereleasing quantal events, as shown after VMAT2 expression in AtT-20 cells. This finding demonstrates that transporter expression can regulate the type of transmitter accumulated in individual vesicles.

It is conceivable that the apparent increase in the frequency of quantal release in VMAT2-overexpressing ventral midbrain neurons is attributable to a large fraction of synaptic vesicles that normally secrete very small amounts of transmitter (i.e., $<1000$ molecules), and that the higher frequency is attributable to an elevated quantal size for this population, allowing them to be distinguished against background noise. It may be that even in mature central catecholaminergic neurons, it is possible that some synaptic vesicles contain no transporter, whereas others contain one or multiple copies. In this case, trafficking of vesicular transporter expression would provide a novel form of synaptic plasticity.

Increased expression of VMAT2 may mimic physiological phenomena in vivo. Under circumstances of high VMAT2 synthesis, the transporter may saturate sorting machinery that determines the protein trafficking to subcellular loci, so that VMAT2 would appear on organelles such as subpopulations of synaptic vesicles, where it otherwise would not be present.

\section{Implications of vesicle transporter regulation in the CNS}

The current findings suggest that one mechanism to segregate transmitter phenotype within a neuron involves altering the local regulated expression of vesicle transporters. Physiological and immunochemical studies in culture (Sulzer et al., 1998) and immunochemical and ultrastructural studies in vivo (Hattori et al., 1991; Sulzer et al., 1998) suggest a partial segregation of dopamine and glutamate release in VTA neurons, so that some axonal varicosities may provide glutamate release but exclude dopamine release. This stands in contrast to the contemporary version of "Dale's principle", which states that an individual neuron releases the same classical transmitter from all of its presynaptic sites (Sossin et al., 1990; Nicoll and Malenka, 1998).

Whereas pharmacological inhibition of vesicular uptake induces a rapid reduction in quantal size in the developing neuromuscular junction (Song et al., 1997), this effect is not obvious in the mature neuromuscular junction until $>100,000$ quanta are released in the presence of the uptake blocker (Van der Kloot et al., 2000). The obvious explanation for this is that there is a very large number of releasable vesicles at the neuromuscular junction, estimated at 170,000-270,000 (Van der Kloot et al., 2000), and so a very large number of vesicles need to release their contents before a decreased mean quantal size becomes measurable. In the CNS, and in dopamine terminals in particular, the number of releasable vesicles are clearly much lower, perhaps 5-100 (Dobrunz and Stevens, 1997). Although the definitions of the vesicle subpopulations may be controversial, the low total number of vesicles present in central presynaptic terminals suggests that regulation of vesicular transport activity would provide rapid and profound changes in transmitter release.

Alterations in quantal release will be particularly important in systems in which extrasynaptic overflow occurs, such as for central dopamine (Garris et al., 1994; Zoli et al., 1998; Sulzer and Pothos, 2000), because postsynaptic receptors will not be saturated and the transmitter released from a quantum encounters multiple postsynaptic elements (Garris et al., 1994; Sulzer and Pothos, 2000). The increased quantal size would provide saturation of dopamine uptake transporters near the site of exocytosis of a synaptic vesicle, leading to a profound increase of temporal and spatial distribution of the released transmitter to multiple postsynaptic sites.

\section{REFERENCES}

Ahnert-Hilger G, Nurnberg B, Exner T, Schafer T, Jahn R (1998) The heterotrimeric $\mathrm{G}$ protein Go2 regulates catecholamine uptake by secretory vesicles. EMBO J 17:406-413.

Alvarez de Toledo G, Fernandez JM (1990) Compound versus multigranular exocytosis in peritoneal mast cells. J Gen Physiol 95:397-409.

Bauerfeind R, Regnier-Vigouroux A, Flatmark T, Huttner WB (1993) Selective storage of acetylcholine, but not catecholamines, in neuroendocrine synaptic-like microvesicles of early endosomal origin. Neuron 11:105-121.

Bellocchio EE, Reimer RJ, Fremeau Jr RT, Edwards RH (2000) Uptake of glutamate into synaptic vesicles by an inorganic phosphate transporter. Science 289:957-960

Bruns D, Jahn R (1995) Real-time measurement of transmitter release from single synaptic vesicles. Nature 377:62-65.

Burke R, Antonelli M, Sulzer D (1998) Glial derived neurotrophic factor prevents apoptosis of substantia nigra neurons during their period of natural cell death. J Neurochem 71:517-525.

Chen TK, Luo G, Ewing AG (1994) Amperometric monitoring of stimulated catecholamine release from rat pheochromocytoma (PC12) cells at the zeptomole level. Anal Chem 66:3031-3035.

Chow RH, von Ruden L (1995) Electrochemical detection of secretion from single cells. In: Single-channel recording (Sakmann B, Neher E, eds), pp 245-276. New York: Plenum.

Ciolkowski EL, Maness KM, Cahill PS, Wightman RM, Evans DH, Fosset B, Amatore C (1994) Disproportionation during electrooxidation of catecholamines at carbon-fiber microelectrodes. Anal Chem 66:3611-3617.

Cubells JF, Rayport S, Rajendran G, Sulzer D (1994) Methamphetamine neurotoxicity involves vacuolation of endocytic organelles and dopaminedependent intracellular oxidative stress. J Neurosci 14:2260-2271.

Dobrunz LE, Stevens CF (1997) Heterogeneity of release probability, facilitation, and depletion at central synapses. Neuron 18:995-1008.

Floor E, Leventhal PS, Wang Y, Meng L, Chen W (1995) Dynamic storage of dopamine in rat brain synaptic vesicles in vitro. J Neurochem 64:689-699.

Fon EA, Pothos EN, Sun BC, Killeen N, Sulzer D, Edwards RH (1997) Vesicular transport regulates monoamine storage and release but is not essential for amphetamine action. Neuron 19:1271-1283.

Garris PA, Ciolkowski EL, Pastore P, Wightman RM (1994) Efflux of dopamine from the synaptic cleft in the nucleus accumbens of the rat brain. J Neurosci 14:6084-6093.

Giorgio R, Brecha N, Edwards RH (1995) Differential expression of two vesicular monoamine transporters. J Neurosci 15:6179-6188.

Harada K, Wu J, Haycock JW, Goldstein M (1996) Regulation of L-DOPA biosynthesis by site-specific phosphorylation of tyrosine hydroxylase in AtT-20 cells expressing wild-type and serine 40-substituted enzyme. J Neurochem 67:629-635.

Hardy S, Kitamura M, Harris-Stansil T, Dai Y, Phipps ML (1997) Construction of adenovirus vectors through Cre-lox recombination. J Virol 71:1842-1849

Hattori T, Takada M, Moriizumi TV, van der Kooy D (1991) Single dopaminergic nigrostriatal neurons form two chemically distinct synaptic types: possible transmitter segregation within neurons. J Comp Neurol 309:391-401.

Hochstetler SE, Puopolo M, Gustincich S, Raviola E, Wightman RM (2000) Real-time amperometric measurements of zeptomole quantities of dopamine released from neurons. Anal Chem 72:489-496.

Horellou P, Guibert B, Leviel V, Mallet J (1989) Retroviral transfer of a human tyrosine hydroxylase cDNA in various cell lines: regulated release of dopamine in mouse anterior pituitary AtT-20 cells. Proc Natl Acad Sci USA 86:7233-7237.

Johnson RG (1988) Accumulation of biological amines into chromaffin granules: a model for hormone and neurotransmitter transport. Physiol Rev 68:232-307.

Kozminski KD, Gutman DA, Davila V, Sulzer D, Ewing AG (1998) Voltammetric and pharmacological characterization of dopamine released from single quantal events in PC12 cells. Anal Chem 70:3123-3130.

Krantz DE, Peter D, Liu Y, Edwards RH (1997) Phosphorylation of a 
vesicular monoamine transporter by casein kinase II. J Biol Chem 272:6752-6759

Kullmann DM, Nicoll RA (1992) Long-term potentiation is associated with increases in quantal content and quantal amplitude. Nature 357:240-244.

Liu Y, Edwards RH (1997) Differential localization of vesicular acetylcholine and monoamine transporters in PC12 cells but not $\mathrm{CHO}$ cells. J Cell Biol 139:907-916.

Liu Y, Schweitzer ES, Nirenberg MJ, Pickel VM, Evans CJ, Edwards RH (1994) Preferential localization of a vesicular monoamine transporter to dense core vesicles in PC12 cells. J Cell Biol 127:1419-1433.

Mena MA, Davila V, Bogaluvsky J, Sulzer D (1998) L-DOPA potentiates NGF-induced outgrowth and quantal dopamine release from PC12 neurites. Mol Pharmacol 54:678-686.

Nakanishi N, Onozawa S, Matsumoto R, Hasegawa H, Yamada S (1995) Cyclic AMP-dependent modulation of vesicular monoamine transport in pheochromocytoma cells. J Neurochem 64:600-607.

Nicoll RA, Malenka RC (1998) A tale of two transmitters. Science 281:360-361.

Nirenberg MJ, Chan J, Liu Y, Edwards RH, Pickel VM (1997) Vesicular monoamine transporter-2: immunogold localization in striatal axons and terminals. Synapse 26:194-198.

Parsons SM, Prior C, Marshall IG (1993) Acetylcholine transport, storage, and release. Int Rev Neurobiol 35:279-390.

Pothos EN, Desmond M, Sulzer D (1996) L-3,4-Dihydroxyphenylalanine increases the quantal size of exocytic dopamine release in vitro. J Neurochem 66:629-636.

Pothos E, Davila V, Sulzer D (1998a) Presynaptic recording of quanta from midbrain dopamine neurons and modulation of the quantal size. J Neurosci 18:4106-4118.

Pothos E, Przedborski S, Davila V, Schmitz Y, Sulzer D (1998b) D2-like dopamine autoreceptor activation reduces quantal size in PC12 cells. J Neurosci 18:5575-5585.

Przedborski S, Khan U, Kostic V, Carlson E, Epstein CJ, Sulzer D (1996) Increased superoxide dismutase activity improves survival of cultured postnatal midbrain neurons. J Neurochem 67:1383-1392.

Rayport S, Sulzer D (1995) Visualization of antipsychotic binding to living mesolimbic neurons reveals D2 receptor mediated, acidotropic and lipophilic components. J Neurochem 65:691-703.

Rayport S, Sulzer D, Shi W-X, Sawasdikosol S, Monaco J, Batson D, Rajendran G (1992) Identified postnatal mesolimbic dopamine neurons in cell culture: morphology and electrophysiology. J Neurosci 12:4264-4280.

Reimer RJ, Fon EA, Edwards RH (1998) Vesicular neurotransmitter transport and the presynaptic regulation of quantal size. Curr Opin Neurobiol 8:405-412.
Song HJ, Ming GL, Fon E, Bellocchio E, Edwards RH, Poo MM (1997) Expression of a putative vesicular acetylcholine transporter facilitates quantal transmitter packaging. Neuron 18:815-826.

Sossin WS, Sweet-Cordero A, Scheller RH (1990) Dale's hypothesis revisited: different neuropeptides derived from a common prohormone are targeted to different processes. Proc Natl Acad Sci USA 87:4845-4848.

Sulzer D, Pothos EN (2000) Presynaptic mechanisms that regulate quantal size. Reviews in the Neurosciences 11:159-212.

Sulzer D, Rayport S (1990) Amphetamine and other psychostimulants reduce $\mathrm{pH}$ gradients in midbrain dopaminergic neurons and chromaffin granules: a mechanism of action. Neuron 5:797-808.

Sulzer D, Chen TK, Lau YY, Kristensen H, Rayport S, Ewing A (1995) Amphetamine redistributes dopamine from synaptic vesicles to the cytosol and promotes reverse transport. J Neurosci 15:4102-4108.

Sulzer D, Joyce MP, Lin L, Geldwert D, Haber SN, Hattori T, Rayport S (1998) Dopamine neurons make glutamatergic synapses in vitro. J Neurosci 18:4588-4602.

Takahashi N, Miner LL, Sora I, Ujike H, Revay RS, Kostic V, JacksonLewis V, Przedborski S, Uhl GR (1997) VMAT2 knockout mice: heterozygotes display reduced amphetamine-conditioned reward, enhanced amphetamine locomotion, and enhanced MPTP toxicity. Proc Natl Acad Sci USA 94:9938-9943.

Travis ER, Wang YM, Michael DJ, Caron MG, Wightman RM (2000) Differential quantal release of histamine and 5-hydroxytryptamine from mast cells of vesicular monoamine transporter 2 knockout mice. Proc Natl Acad Sci USA 97:162-167.

Van der Kloot W (1991) The regulation of quantal size. Prog Neurobiol 36:93-130.

Van der Kloot WV, Colasante C, Cameron R, Molgo J (2000) Recycling and refilling of transmitter quanta at the frog neuromuscular junction. J Physiol (Lond) 523:247-258.

Wang YM, Gainetdinov RR, Fumagalli F, Xu F, Jones SR, Bock CB, Miller GW, Wightman RM, Caron MG (1997) Knockout of the vesicular monoamine transporter 2 gene results in neonatal death and supersensitivity to cocaine and amphetamine. Neuron 19:1285-1296.

Wightman RM, Jankowski JA, Kennedy RT, Kawagoe KT, Schroeder TJ, Leszczyszyn DJ, Near JA, Diliberto Jr EJ, Viveros OH (1991) Temporally resolved catecholamine spikes correspond to single vesicle release from individual chromaffin cells. Proc Natl Acad Sci USA 88:10754-10758.

Zoli M, Torri C, Ferrari R, Jansson A, Zini I, Fuxe K, Agnati LF (1998) The emergence of the volume transmission concept. Brain Res Brain Res Rev 26:136-147. 